\title{
ESTUDIO COMPARATIVO DE LOS MÉTODOS ESPECTROFOTÓMETRICO Y POTENCIOMÉTRICO PARA LA DETERMINACIÓN CUANTITATIVA DE FENOLES TOTALES EN PLANTAS MEDICINALES ECUATORIANAS
}

\author{
COMPARATIVE STUDY OF THE METHODS \\ SPECTROPHOTOMETRIC AND POTENTIOMETRIC \\ FOR QUANTITATIVE DETERMINATION OF TOTAL PHENOLS \\ IN ECUADORIAN MEDICINAL PLANTS
}
Anyi Jaramillo Jiménez' , Carmita Jaramillo Jaramillo \& Luisa Rojas de Astudillo ${ }^{2 *}$

Recibido: 8 de noviembre 2021 / Aceptado: 7 de enero 2022 DOI 10.26807/ia.v10i1.228

Palabras clave: fenoles; Folinn y Ciocalteau; plantas medicinales; titulación potenciométrica.

Keywords: Folinn and Ciocalteau, medicinal plants, phenols, potentiometric titration.

1Universidad Técnica de Machala, Machala, Ecuador. (anyisssita2203@gmail.com, cjaramillo @utmachala.edu.ec)

2 Universidad de Oriente, Cumana, Venezuela, (* correspondencia: Irojas40@yahoo.com) 


\section{RESUMEN}

En este estudio, fueron comparados los valores de las concentraciones de fenoles totales en extractos acuosos de plantas medicinales ecuatorianas que fueron obtenidos usando el método espectrofotométrico desarrollado por Folinn y Ciocalteau y por titulación potenciométrica. Por el método potenciométrico se cuantificaron los fenoles totales presentes en cuatro plantas medicinales (Ageratum conyzoides, Cnidoscolus chayamansa, Cynara scolymus y Taraxacum officinale), las cuales fueron seleccionadas por ser representativas de los contenidos alto, medio, intermedio y bajo de fenoles totales de las doce plantas previamente analizadas espectrofotométricamente. Los resultados de la cuantificación de fenoles totales por titulación potenciométrica tuvieron una alta correlación $\left(\mathrm{R}^{2}=0,987\right)$ con los valores obtenidos por el método espectrofotométrico, a las concentraciones de 0,70 a 24,0 mg/g. El uso de la potenciometría para cuantificar fenoles totales resultó amigable con el ambiente al utilizar concentraciones muy diluidas de reactivos y reemplazar las substancias tóxicas empleadas habitualmente en los métodos espectrofotométricos.

\section{ABSTRACT}

In this study, the values of total phenol concentrations in aqueous extracts of ecuadorian medicinal plants, obtained using the spectrophotometric method developed by Folinn and Ciocalteau and by the potentiometric titration method, were compared. By the potentiometric method, the total phenols present in four medicinal plants (Ageratum conyzoides, Cnidoscolus chayamansa, Cynara scolymus y Taraxacum officinale) were quantified, which were selected for being representatives of the high, medium, intermediate, and low contents of total phenols of the twelve plants previously analyzed spectrophotometrically. The results of the quantification of total phenols by potentiometric titration had a high correlation $\left(R^{2}=0.987\right)$ with the values obtained by the spectrophotometric method, at concentrations of 0.70 to $24.0 \mathrm{mg} / \mathrm{g}$. The use of potentiometry to quantify total phenols was environmentally friendly by using very dilute concentrations of reagents and replacing the toxic substances commonly used in spectrophotometric methods. 


\section{INTRODUCCIÓN}

Los metabolitos secundarios en plantas con mayores potenciales antioxidantes son los compuestos fenólicos y se pueden dividir en cinco grupos generales, a saber, los ácidos fenólicos, los flavonoides, los lignanos, los estilbenos y los taninos (Duthie et al. 2000; Salehi et al., 2020). Dado que el consumo de dietas ricas en compuestos fenólicos se correlaciona con una reducción de las enfermedades coronarias, asociándose con mayores expectativas de vida, actualmente, hay un interés creciente por estos compuestos. Además, dichos compuestos presentan varias actividades biológicas, entre ellas, antimicrobiana, antiviral, antiinflamatoria, hepatoprotectoras y anticancerígenas (Nijveldt et al., 2001; Serrano et al., 2009; Liu et al., 2019).

En la naturaleza, los fenoles se encuentran mayormente en forma de glucósidos, por lo que son solubles en agua y solventes orgánicos. También pueden encontrarse unidos a ácidos carboxílicos, ácidos orgánicos, aminas, lípidos y a otros compuestos fenólicos (Bravo, 1998).
Para la determinación cuantitativa de fenoles totales, el método espectrofotométrico desarrollado por Folinn y Ciocalteau (FC) (Folinn \& Ciocalteau, 1927) es el más usado, el cual utiliza la reacción entre grupos hidroxilo de los fenoles con un reactivo de fosfomolibdato (mezcla de ácidos fosfowolfrámico y fosfomolibdíco en medio básico), el cual se reduce al oxidar los compuestos fenólicos, originando óxidos azules de wolframio $\left(\mathrm{W}_{8} \mathrm{O}_{23}\right)$ y molibdeno $\left(\mathrm{Mo}_{8} \mathrm{O}_{23}\right)$, para cuantificar el contenido de fenoles totales (Singleton et al., 1999; De Beer et al., 2004). Si bien esta es una técnica analítica conveniente y simple para determinar cuantitativamente fenoles totales en diversas muestras, entre ellas vinos y extractos vegetales, tiene las desventajas de no usar reactivos ecológicos y de precisar de un prolongado tiempo de procesamiento (Sun et al., 2019); además, las proteínas tienen una alta influencia en los valores de fenoles totales, requiriendo enmascaramiento de esa interferencia, para obtener el real contenido de fenoles en las muestras analizadas (Escarpa \& González, 2001). 
Aparte del método Folinn Ciocalteau, se han utilizado otros métodos analíticos para determinar el contenido fenólico, entre ellos la cromatografía líquida (Mizzi et al., 2020), los ensayos de voltamperometría, de polarografía, valoración culombimétrica y potenciométrica, usando diversos electrodos (Wang \& Li, 1989; Rodríguez-Méndez et al., 2008; Elbehery et al., 2019).

Los fenoles son mucho más ácidos que los alcoholes, por lo que pueden reaccionar con hidróxido de sodio acuoso a bajas concentraciones y se pueden obtener las constantes de disociación a partir de una titulación potenciométrica de soluciones de fenoles con soluciones de $\mathrm{NaOH}$ (Mota et al., 2008). De los análisis de Huma et al. (1999) fue determinado el pKa para fenol, siendo pKa $=9,989$ a $20{ }^{\circ} \mathrm{C}$. Esto permite que por medio de las curvas potenciométricas se determinen las concentraciones de fenoles provenientes de una reacción con hidróxido de sodio. Debido a que se usan concentraciones diluidas, esta metodología electroquímica va de la mano con la química verde y con el cuidado del ambiente. Además, presentan ventajas tales como disponibilidad, rapidez, baja costos de equipos, pocos reactivos, y procedimientos de medición sencillos (Hoyos et al., 2017; De Leite et al., 2018). Es por tal razón que en esta investigación se compararon los métodos potenciométricos y espectrofotomé trico, para la determinación cuantitativa de fenoles totales en especies vegetales ecuatorianas.

\section{MATERIALES Y MÉTODOS}

\section{Plantas medicinales}

Se recolectaron muestras representativas de doce especies de plantas medicinales: a) Bougainvillea spectabilis, Cnidoscolus chayamansa, Parthenium hysterophorus y Piper carpunya en la provincia El Oro (3 ${ }^{\circ}$ $\left.19^{\prime} 36.84^{\prime \prime} \mathrm{S}-79^{\circ} 48^{\prime} 17.64^{\prime \prime} \mathrm{W}\right)$; b)
Cecropia peltata y Artemisa vulgaris en Calichana $\left(3^{\circ} 22^{\prime} 07,2^{\prime \prime}\right.$ S, $79^{\circ} 48^{\prime} 32,9^{\prime \prime}$ W), Provincia El Oro; c) Taraxacum officinale en la provincia Pichincha ( $0^{\circ} 122.8^{\prime \prime} \mathrm{N}-78^{\circ} 53^{\prime}$ 31.2" W) y d) Cynara scolymus, Linum usitatissimum, Parietaria officinalis, Ageratum conyzoides y Bo- 
rrago officinalis fueron compradas en estado fresco en el mercado de Machala, Machala, Ecuador.

De cada planta se utilizaron sólo las hojas, que fueron lavadas con agua destilada y escurridas en un secador artesanal abierto, por 24 horas, a la temperatura $\left(22 \pm 2{ }^{\circ} \mathrm{C}\right)$ del laboratorio. Posteriormente, fueron secadas en una estufa (MEMMERT SNB 400 con flujo de aire) a $37^{\circ} \mathrm{C}$ por 24 horas. Seguidamente, se pulverizaron con un molino (Lab. Mill serial No. 56969, Type AR 400 Erweka®, Germany) y se tamizaron a $\leq 1 \mathrm{~mm}$.

\section{Extracto acuoso}

Para preparar el extracto acuoso, $15 \mathrm{~g}$ del material vegetal molido y seco se sumergieron en $150 \mathrm{~mL}$ de agua destilada caliente, a $80{ }^{\circ} \mathrm{C}$, hasta un tiempo máximo de una hora, después de lo cual se filtró (WHO 2011). La misma solución del extracto de cada planta se usó el mismo día para las determinaciones cuantitativas espectrofotométricas y potenciométricas.

\section{Determinación de fenoles por téc- nica espectrofotométrica}

Se utilizó el método sugerido por Velásquez (2004) y Blainski et al.
(2013). La curva de calibración se preparó a partir de una serie de soluciones patrón de ácido gálico (Sigma-Aldrich, Alemania), con concentraciones de 0, 10, 20, 30, 40 y $50 \mathrm{mg} / \mathrm{L}$. Para el análisis de la muestra, $2 \mathrm{~mL}$ del extracto acuoso, previamente diluidos 1:50 con etanol, fueron mezclados con $1 \mathrm{~mL}$ del reactivo de Folinn Ciocalteu (SigmaAldrich, Alemania), se agitó y se dejó en reposo durante cinco minutos. Luego se le añadieron $0,5 \mathrm{~mL}$ de carbonato de sodio al $5 \%$, la solución se agitó y se llevó a un volumen de $10 \mathrm{~mL}$ con agua destilada. Después de 30 minutos de reacción, se hicieron las determinaciones espectrofotométricas a 700 nm (Velásquez, 2004) en un espectrofotómetro (Evolution 201, Thermo Scientific). Todos los análisis fueron hechos por triplicado. Los resultados fueron expresados como mg equivalentes a ácido gálico/g de material seco de planta.

\section{Determinación de fenoles por po- tenciometría}

Para la determinación de fenoles totales por potenciometría se seleccionaron cuatros plantas por ser representativas de los contenidos alto ( $T$. officinale), intermedio alto 
(A. conyzoides), intermedio bajo (C. chayamansa) y el más bajo (C. scolymus) de fenoles totales de las doce especies vegetales analizadas previamente por espectrofotometría. Se usó el ácido gálico como estándar.

El método usado fue una modificación del producido por Huma et al. (1999), la cual consistió en usar el método de adición de analito, en este caso, un $\mathrm{mL}$ del extracto acuoso de la planta medicinal se agregó a 25 mL de ácido gálico (20 mg/L), logrando así una solución que fue valorada con una solución de $\mathrm{NaOH}$ (0,05 mol/L), agregando cada vez un volumen de $50 \mu \mathrm{L}$ y midiendo el $\mathrm{pH}$ simultáneamente, utilizando un medidor de $\mathrm{pH}$ digital (OAKTON, 2700). El sistema de medición fue calibrado con tampones de $\mathrm{pH}$ 4,00 y 7,00 de Merck, trazables a materiales de referencia estándar de NIST. A partir de las curvas derivativas de la valoración potenciométrica se determinó el punto de equivalencia de la reacción acido-base. El ácido gálico de concentración 20 mg/L y un volumen de $25 \mathrm{~mL}$ fue usado como sustancia de referencia en la calibración electroquímica para la cuantificación de fenoles.

Los cálculos se realizaron con los valores diferenciales $\mathrm{pH}, \Delta \mathrm{pH}$, $\mathrm{V}(\mathrm{mL}), \log \mathrm{V}, \Delta \log (\mathrm{V}), \Delta \mathrm{pH} / \Delta \log (\mathrm{V})$, se usó la relación $\log (\mathrm{V})$ versus $\Delta \mathrm{pH}$ $/ \Delta \log (\mathrm{V})$ para obtener el punto de equivalencia y determinar la concentración de fenoles en las muestras de plantas medicinales.

\section{RESULTADOS}

En la curva de calibración se obtuvo una linealidad con un $\mathrm{R}^{2}=0,997$. El producto de la reacción de los fenoles con el reactivo Folinn-Ciolcateu es estable hasta los 60 minutos. Esta técnica permitió la cuantificación de los fenoles presentes en las distintas especies vegetales analizadas.
Como lo indica la Tabla 1, el contenido de fenoles en las plantas medicinales osciló entre los valores, 22,3 mg equivalentes de A.G/g de material seco y $0,90 \mathrm{mg}$ equivalentes de A.G/g de material seco para las plantas T. officinale y C. scolymus, respectivamente. 
Tabla 1. Contenido de fenoles en las especies de plantas medicinales obtenido por el método espectrofotométrico

\begin{tabular}{lc}
\hline $\begin{array}{l}\text { Plantas } \\
\text { medicinales }\end{array}$ & $\begin{array}{c}\text { Concentración } \\
\text { (mg/g) }\end{array}$ \\
\hline T. officinale & $22,30 \pm 0,03$ \\
B. spectabilis & $8,50 \pm 0,01$ \\
P. hysterophorus & $6,00 \pm 0,02$ \\
A. conyzoides L & $5,60 \pm 0,04$ \\
L. sitatissimum & $4,70 \pm 0,05$ \\
B. officinalis & $3,90 \pm 0,01$ \\
C. chayamansa & $3,30 \pm 0,02$ \\
P. officinalis & $2,80 \pm 0,03$ \\
P. carpunya & $2,80 \pm 0,04$ \\
A. cumanensi & $2,40 \pm 0,03$ \\
C. obtusifolia & $1,70 \pm 0,04$ \\
C. scolymus & $0,90 \pm 0,02$ \\
\hline
\end{tabular}

En la Tabla 2 se muestran los valores de $\mathrm{pH}, \mathrm{V}(\mathrm{mL}), \log \mathrm{V}$ y $\Delta \mathrm{pH} / \Delta \log (\mathrm{V})$ obtenidos con el ácido gálico (20 $\mathrm{mg} / \mathrm{L})$ y el valorante $\mathrm{NaOH}(0,05$ $\mathrm{mol} / \mathrm{L})$, mostrando que en la relación $\Delta \mathrm{pH} / \Delta \log (\mathrm{V})$ el punto 9,38 corresponde al $\mathrm{pH} 9,60$, y a un volumen $0,45 \mathrm{~mL}$ de la solución de $\mathrm{NaOH}$, indicando el punto al que reaccionan los fenoles totales con el $\mathrm{NaOH}$, por lo que es el punto de equivalencia de la reacción de $\mathrm{NaOH}$ con el ácido gálico.
Tabla 2. Valores obtenidos de la valoración potenciométrica de $25 \mathrm{~mL}$ de ácido gálico $(20 \mathrm{mg} / \mathrm{L})$ Vs. $\mathrm{NaOH}(0,05 \mathrm{~mol} / \mathrm{L})$

\begin{tabular}{cccc}
\hline $\mathrm{pH}$ & $\begin{array}{c}\mathbf{V} \\
(\mathrm{ml})\end{array}$ & $\begin{array}{c}\text { Log } \\
(\mathrm{V})\end{array}$ & $\begin{array}{c}\Delta \mathrm{pH} / \\
\Delta \log (\mathrm{V})\end{array}$ \\
\hline 8,72 & 0,35 & $-0,46$ & 1,79 \\
9,12 & 0,40 & $-0,40$ & 6,90 \\
9,60 & 0,45 & $-0,35$ & 9,38 \\
9,87 & 0,50 & $-0,30$ & 5,90 \\
10,0 & 0,55 & $-0,26$ & 4,11 \\
10,2 & 0,60 & $-0,22$ & 4,50 \\
10,3 & 0,65 & $-0,19$ & 3,16 \\
\hline
\end{tabular}

En la Tabla 3 se muestran los valores diferenciales $\mathrm{pH}, \mathrm{V}(\mathrm{mL}), \log (\mathrm{V}) \mathrm{y}$ $\Delta \mathrm{pH} / \Delta \log (\mathrm{V})$ obtenidos con la mezcla de $25 \mathrm{~mL}$ de ácido gálico (20 $\mathrm{mg} / \mathrm{L}$ ) y $1 \mathrm{~mL}$ del extracto acuoso de $T$. officinale frente al valorante $\mathrm{NaOH}(0,05 \mathrm{~mol} / \mathrm{L})$. Se observa que al valor de 8,15 de la relación $\Delta \mathrm{pH} / \Delta \log \mathrm{V}$ corresponde al de 9,68 del $\mathrm{pH}$, al volumen de $1,50 \mathrm{~mL}$ del titulante $(\mathrm{NaOH} 0,05 \mathrm{~mol} / \mathrm{L})$, es el punto de mayor inflexión, distinguido en la Figura 1, siendo el de equivalencia de la reacción entre los fenoles presentes en $T$. officinale y en el ácido gálico frente a $\mathrm{NaOH}(0,05$ $\mathrm{mol} / \mathrm{L})$. 
Tabla 3. Valores obtenidos en la valoración potenciométrica de la mezcla de $25 \mathrm{~mL}$ de ácido gálico (20 mg/L) y $1 \mathrm{~mL}$ del extracto acuoso de $T$. officinale $\mathrm{Vs} \mathrm{NaOH}(0,05 \mathrm{~mol} / \mathrm{L})$

\begin{tabular}{cccc}
\hline pH & $\begin{array}{c}\mathbf{V} \\
(\mathrm{ml})\end{array}$ & $\begin{array}{c}\text { Log } \\
(\mathrm{V})\end{array}$ & $\begin{array}{c}\Delta \mathrm{pH} / \\
\Delta \log (\mathrm{V})\end{array}$ \\
\hline 9,52 & 1,40 & 0,15 & 3,17 \\
9,56 & 1,45 & 0,16 & 2,62 \\
$\mathbf{9 , 6 8}$ & 1,50 & 0,18 & $\mathbf{8 , 1 5}$ \\
9,72 & 1,55 & 0,19 & 2,81 \\
9,76 & 1,60 & 0,20 & 2,90 \\
9,80 & 1,65 & 0,22 & 2,99 \\
\hline
\end{tabular}

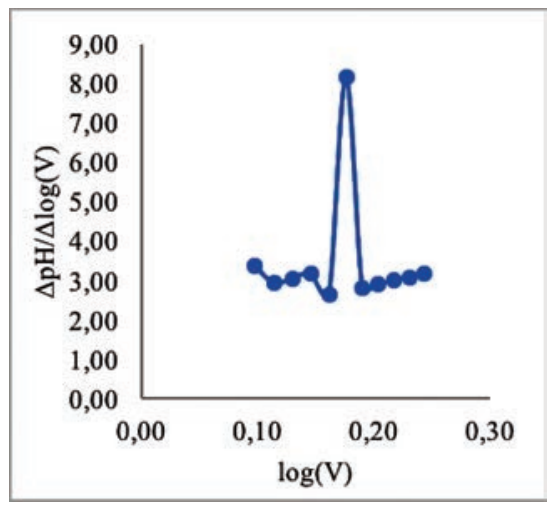

Figura 1. Curva de la valoración electroquímica para la reacción de fenoles de la mezcla de ácido gálico y extracto de $T$. officinale con $\mathrm{NaOH}(0,05 \mathrm{~mol} / \mathrm{L})$

Los valores de las concentraciones de fenoles totales obtenidos por medio del metodo potenciométrico oscilaron entre 23,8 mg equivalentes de A.G/g de extracto y $0,78 \mathrm{mg}$ equivalente de A.G/g de extracto, para las plantas $T$. officinale y $C$. scolymus, respectivamente.

A continuación, la Figura 2 permite apreciar que no hubo diferencias significativas entre los valores de las concentraciones de fenoles totales obtenidos por las dos técnicas empleadas (potenciometría y espectrofotometría). La gráfica muestra una alta regresión lineal con $\mathrm{R}^{2}$ de 0,987, indicando que el método potenciométrico propuesto es un método confiable para determinar fenoles totales en especies vegetales y similar al método espectrofotométrico.

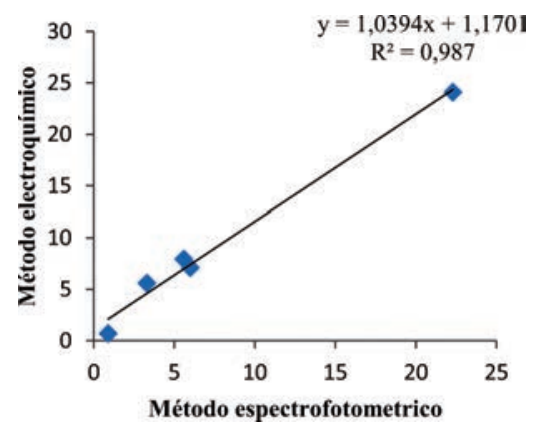

Figura 2. Análisis de regresión entre los valores de fenoles totales para cada planta medicinal por los métodos espectrofotométrico y potenciométrico 


\section{DISCUSIÓN}

En cuanto a las concentraciones de fenoles totales, los valores más altos fueron encontrados en la planta medicinal T. officinale, los cuales fueron similares a los obtenidos por Makkar et al. (2009) y un poco más alto que los encontrados por Hudec et al. (2007), Sengul et al. (2009) y Ghaima et al. (2013).

La técnica de titulación potenciométrica para la cuantificación de fenoles en extractos acuosos de especies vegetales resultó ser un método preciso a concentraciones del orden de $\mathrm{mg} / \mathrm{L}$, no se detectaron efectos de dilución a esa concentración. Los resultados coinciden por lo expresado por Huma et al. (1999): Convencionalmente los datos de titulación a partir de la gráfica del $\mathrm{pH}$ frente al volumen de titulante para obtener una curva sigmoide que por simetría ayuda a localizar el punto de equivalencia en un apropiado valor de $\mathrm{pH}$. Sin embargo, a medida que la naturaleza de los ácidos y las bases que interactúan cambia de fuerte a débil esa curva de titulación no es precisa para identificar el punto de equivalencia.

El caso de la titulación de fenol contra hidróxido de sodio es típico de un ácido muy débil que se titula contra una base fuerte, por lo que se hizo evidente que la curva de titulación $\mathrm{pH}$ frente al volumen $\mathrm{V}$ no tiene punto de inflexión, la cual no es útil para determinaciones cuantitativas. Sin embargo, cuando las gráficas fueron de los diferenciales $\Delta \mathrm{pH} / \Delta \log (\mathrm{V})$ versus $\log (\mathrm{V})$, el punto de equivalencia fue muy nítido y proporciona con precisión el volumen de titulante $(\mathrm{NaOH} 0,05 \mathrm{~mol} / \mathrm{L})$ usado, para luego determinar las concentraciones de fenoles totales en las plantas medicinales, las cuales fueron corroboradas por las altas correlaciones positivas con los valores de fenoles totales obtenidos espectrofotométricamente. 


\section{CONCLUSIÓN}

La alta correlación lineal con $\mathrm{R}^{2}$ de 0,987 entre los métodos espectrofotométrico y potenciométrico indican que el método potenciométrico propuesto, con adición de analito, es confiable para la determinación cuantitativa de fenoles en el intervalo de las concentraciones estudiadas, de 0,70 a 24,0 mg/g. Además, el uso de la potenciometría para la cuantificación de fenoles totales resulta amigable con el ambiente, debido a que se utilizan concentraciones muy diluidas de reactivos que son menos tóxicos que los empleados habitualmente en los métodos espectrofotométricos.

\section{AGRADECIMIENTOS}

Los autores agradecen al Proyecto Prometeo de la Secretaría Nacional de Educación Superior, Ciencia y
Tecnología de la República de Ecuador (SENESCYT), por el financiamiento de esta investigación.

\section{LISTA DE REFERENCIAS}

Blainski, A., Lopes G. \& Palazzo de Mello, J. (2013). Application and analysis of the folin ciocalteu method for the determination of the total phenolic content from Limonium brasiliense L. Molecules, 18(6), 6852-6865. doi:10.3390/molecules 18066852

Bravo, L. (1998). Poliphenol: chemistry, dietary sources, metabolism, and nutritional significance. Nutrition Reviews, 56, 317-333. doi: 10.1111/j.1753-4887.1998. tb01670.x.

De Beer, D., Harbertson, J.F., Kilmartin, P.A., Roginsky, V., Barsukova, T., Adams, D.O., $\&$ Waterhouse, A.L. (2004). Phenolics: A comparison of diverse analytical methods. American Journal of Enology and Viticulture, 55, 389-400. 
De Leite, K.C.S., Garcia, L.F., Lobón, G.S. \& et al. (2018). Antioxidant activity evaluation of dried herbal extracts: an electroanalytical approach. Brazilian Journal of Pharmacognosy, 28, 325-332. https://doi.org/10.1016/j.bjp.2018.04.004.

Duthie, G.G., Duthie, S.J., \& Kyle, J.A.M. (2000). Plant polyphenols in cancer and heart disease: Implications as nutritional antioxidants. Nutrition Research Reviews, 13(1),79-106. DOI: 10.1079/095442200108729016.

Elbehery, N.H.A., Amr, A.E.-G.E., Kamel, A.H., Elsayed, E.A. \& Hassan, S.S. (2019). Novel potentiometric 2,6-dichlorophenolindo-phenolate (DCPIP) membranesased Sensors: Assessment of their Input in the determination of total phenolics and ascorbic acid in beverages. Sensors, 19(9), 2058. https://doi.org/10.3390/ s19092058.

Escarpa, A. \& González, M.C. (2001) Approach to the content of total extractable phenolic compounds from different food samples by comparison of chromatographic and spectrophotometric methods. Analytica Chimica Acta, 427, 119-127. http://dx.doi.org/10.1016/S0003-2670(00)01188-0.

Folinn, C., \& Ciocalteau, V. (1927). Tyrosine and tryptophan determination in proteins. The Journal of the biological Chemistry, 73, 627-650.

Ghaima, K. K., Hashim, N. M., \& Ali, S. A. (2013). Antibacterial and antioxidant activities of ethyl acetate extract of nettle (Urtica dioica) and dandelion (Taraxacum officinale). Journal of Applied Pharmaceutical Science, 3, 96-99. doi:10.7324 /JAPS.2013.3518

Hoyos, J., Vázquez, M., \& Contreras-Calderón, J. (2017). Electrochemical methods as a tool for determining the antioxidant capacity of food and beverages: A review. Food Chemistry, 221, 1371- 1381. https://doi.org/10.1016/j.foodchem. 2016.11.017

Hudec, J, Ria Burdovaä M., Kobida, L. Komora L., Macho V., Kogan G., Turianica I., \& et al. (2007). Antioxidant Capacity Changes and Phenolic Profile of Echinacea purpurea, Nettle (Urtica dioica L.), 1 and Dandelion (Taraxacum officinale) after Application of Polyamine and Phenolic Biosynthesis Regulators. Journal of Agricultural and Food Chemistry, 55(14), 5689-96. doi:10.1021/jf070777c 
Huma F, Jaffar M, \& Masud K. (1999). A Modified potentiometric method for the estimation of phenol in aqueous systems. Turkish Journal of Chemistry, 23, 415-422.

Liu, J., Yong, H., Yao, X., Hu, H., Yun, D., \& Xiao, L. (2019). Recent advances in phenolic-protein conjugates: synthesis, characterization, biological activities and potential applications RSC Advances, 9, 35825-35840. https://doi.org/10.1039/ C9RA07808H

Makkar, H. P., Norvsambuu, T., Lkhagvatseren, S., \& Becker, K. (2009). Plant secondary metabolites in some medicinal plants of Mongolia used for enhancing animal health and production. Tropicultura, 27, 159-167. doi: 10.3390/genes9060309.

Martínez- Valverde, I., Periago, M., \& Ros, G. (2000). Significado nutricional de los compuestos fenólicos de la dieta. Archivos Latinoamericanos de Nutrición, 50(1), 5-18.

Mizzi, L., Chatzitzika, C., Gatt, R., \& Valdramidis, V. (2020). HPLC analysis of phenolic compounds and flavonoids with overlapping peaks. Food technology and biotechnology, 589(1), 12-19. https://doi.org/10.17113/ftb.58.01.20.6395.

Mota, F., Queimada, A., Pinho, S., \& Macedo. E. (2008). Aqueous solubility of some natural phenolic compounds. Industrial and Engineering Chemistry Research, 47(15), 5182-5189. https://doi.org/10.1021/ie071452o

Nijveldt, R.J., Van Nood, E., Van Hoorn, D.E.C., Boelens, P.G., \& Van Norren, K. (2001). Flavonoids: A review of probable mechanisms of action and potential applications. The American Journal of Clinical Nutrition, 74, 418-425. https://doi.org/10. 1093/ajcn/74.4.418

Rodríguez-Méndez, M., Apetrei, C., \& De Saja, J. (2008). Evaluation of the polyphenolic content of extra virgin olive oils using an array of voltammetric sensors. Electrochimica Acta, 53, 5867-5872. https://doi.org/10.1016/j.electacta .2008.04.006

Salehi, B., Azzini E., Zucca P., Varoni E., Anil Kumar N., Luciana Dini L. \& et al. (2020). Plant-derived bioactives and oxidative stress-related disorders: A key trend towards healthy aging and longevity promotion. Applied Science, 10, 947; doi:10.3390/app10030947. 
Sengul, M., Yildiz, H., Gungor, N., Cetin, B., Eser, Z., \& Ercisli, S. (2009). Total phenolic content, antioxidant and antimicrobial activities of some medicinal plants. Pakistan Journal of Pharmaceutical Sciences, 22, 102-106.

Serrano, J., Puupponen-Pimiä, R., Dauer, A., Aura, A.M., \& Saura-Calixto, F. (2009). Tannins: Current knowledge of food sources, intake, bioavailability, and biological effects. Molecular Nutrition \& Food Research, 53, 310-329. DOI: 10.1002/mnfr.200900039

Singleton, V.L., Orthofer, R., \& Lamuela-Raventós, R.M (1999). Analysis of total phenols and other oxidation substrates and antioxidants by means of Folin-Ciocalteu reagent. Methods in Enzymology, 299, 152-178. https://doi.org/10.1016/S00766879(99)99017-1

Sun, Z., Zhang, Y., Xu, X., Wang, M., \& Kou L. (2019). Determination of the total phenolic content in wine samples using potentiometric method based on permanganate ion as an indicator. Molecules, 24(18), 3279. https://doi.org/10.3390/ molecules24183279.

Velásquez A. (2004). Extracción de taninos presentes en el banano verde. Revista Lasallista de Investigación, 1(2), 17-22.

Wang, J. \& Li, R. (1989). Highly stable voltametric measurements of phenolic compounds at poly(3-methylthiophene)-coated glassy carbon electrodes. Analytical Chemistry, 61, 2809-2811. https://doi.org/10.1021/ac00199a025

World Health Organization (WHO) (2011). Quality control methods for medicinal plant materials. WHO Pres. Geneva. p 1-4, 26-31. 